\title{
180.
}

\section{NOTE ON THE REDUCTION OF AN ELLIPTIC ORBIT TO A FIXED PLANE.}

[From the Quarterly Mathematical Journal, vol. II. (1858), pp. 49-54.]

THE principal object of the present Note is to obtain an expression for the quantity $\epsilon_{0}$ which I call the modified mean longitude at epoch, viz. taking as the elements the longitude of the node, inclination and any four elements which determine the motion in the plane of the orbit, then the longitude measured in the fixed plane (or reduced longitude) will be a function of the form

$$
n t+\epsilon_{0}+\text { periodic terms, }
$$

where $\epsilon_{0}$ is a determinate function of the elements, and it is proposed to find the expression of this function. But as the corresponding formulæ relating to the eccentricity and longitude of the pericentre are not in general given as part of the theory of elliptic motion, but occur only, so far as I am aware, in works on the lunar theory, I have thought it desirable to include these formulæ and take as the subject of this Note the reduction of an elliptic orbit to a fixed plane. Write

$a_{1}$, the semiaxis-major,

$e_{1}$, the eccentricity $\left(=\sin \kappa_{1}\right)$,

$\varpi_{1}$, the longitude of pericentre in orbit,

$\epsilon_{1}$, the mean longitude in orbit at epoch,

$\theta$, the longitude of node,

$\phi$, the inclination $\left(=\tan ^{-1} \gamma\right)$,

and moreover

$$
n_{1} \text {, the mean motion }\left\{=\sqrt{ }\left(\frac{\sigma}{a_{1}^{3}}\right)\right\} \text {, }
$$


where by longitude in orbit is to be understood as usual a longitude measured in the fixed plane as far as the node and from the node in the plane of the orbit: the meaning of $\epsilon_{1}$ is perhaps more clearly fixed by saying that $\epsilon_{1}-\sigma_{1}$ denotes the mean anomaly at epoch.

The elements most nearly corresponding to the above, in the orbit reduced to the fixed plane, are

$a_{0}$, the modified semiaxis-major,

$e_{0}$, the modified eccentricity,

$\varpi_{0}$, the modified longitude of pericentre,

$\epsilon_{0}$, the modified mean longitude at epoch,

$\theta$, the longitude of node,

$\phi$, the inclination $\left(=\tan ^{-1} \gamma\right)$,

and moreover

$n$, the mean motion $\left\{\right.$ not equal to $\left.\sqrt{ }\left(\frac{\sigma}{a_{0}^{3}}\right)\right\}$,

where $\theta, \phi, n$ are the same as in the actual orbit, but $a_{0}, e_{0}, \varpi_{0}, \epsilon_{0}$ are defined as follows: viz. $e_{0}, \varpi_{0}$ are functions of $e_{1}, \varpi_{1}, \theta, \phi$ given by the equations

$$
\begin{aligned}
\tan \left(\varpi_{0}-\theta\right) & =\sec \phi \tan \left(\varpi_{1}-\theta\right), \\
e_{0} & =\frac{e_{1} \cos \left(\varpi_{1}-\theta\right)}{\cos \left(\varpi_{0}-\theta\right)},
\end{aligned}
$$

$a_{0}$ is determined by the condition

$$
a_{0}\left(1-e_{0}^{2}\right)=a_{1}\left(1-e_{1}^{2}\right),
$$

and $\epsilon_{0}$ is determined so that the reduced longitude may be equal to

$$
n t+\epsilon_{0}+\text { periodic terms. }
$$

It is easy to see that considering the orbit and the fixed plane as great circles of the sphere, and projecting the pericentre upon the fixed plane by an arc perpendicular to the orbit, then $\varpi_{0}$ denotes the longitude of such projection of the pericentre; and $e_{0}$ is equal to $e_{1}$ into the secant of the projecting arc. In fact we have a rightangled spherical triangle, of which the projecting arc in question is the perpendicular, and the hypothenuse and base are $\varpi_{0}-\theta$ and $\varpi_{1}-\theta$ respectively, and the base angle is the inclination $\phi$. It is to be remarked that $\varpi_{0}$ is not the reduced longitude of the pericentre, an expression that would signify the longitude of the projection of the pericentre by an arc perpendicular to the fixed plane; this is the reason why I have throughout used the word modified instead of what would at first sight have appeared the natural one, viz. the word reduced. The modified semiaxis-major is obviously a semiaxis-major calculated from the latus rectum of the orbit by means of the modified ècentricity $e_{0}$. 
The relations between $e_{0}, \varpi_{0}, e_{1}, \varpi_{1}$ may be written

or again

$$
\begin{aligned}
\tan \left(\varpi_{0}-\theta\right) & =\sec \phi \tan \left(\varpi_{1}-\theta\right), \\
e_{0} & =e_{1} \sec \phi \sqrt{ }\left\{1-\sin ^{2} \phi \sin ^{2}\left(\varpi_{1}-\theta\right)\right\},
\end{aligned}
$$

$$
\begin{aligned}
\tan \left(\varpi_{1}-\theta\right) & =\cos \phi \tan \left(\varpi_{0}-\theta\right), \\
e_{1} & =e_{0} \sqrt{ }\left\{1-\sin ^{2} \phi \sin ^{2}\left(\varpi_{0}-\theta\right)\right\} .
\end{aligned}
$$

Write now

$r_{1}$, the radius vector,

$v_{1}$, the longitude in orbit,

$\lambda$, the latitude $\left(=\tan ^{-1} s\right)$,

and in like manner

$r_{0}$, the reduced radius vector,

$v_{0}$, the reduced longitude,

$\lambda$, the latitude $\left(=\tan ^{-1} s\right)$.

Then $v_{1}-\theta$ and $v_{0}-\theta$ are the hypothenuse and base of a right-angled spherical triangle, the perpendicular being $\lambda$ and the angle at the base being $\phi$. We have

$$
\begin{aligned}
\tan \lambda & =\tan \phi \sin \left(v_{0}-\theta\right), \\
\sin \lambda & =\sin \phi \sin \left(v_{1}-\theta\right), \\
\tan \left(v_{0}-\theta\right) & =\cos \phi \tan \left(v_{1}-\theta\right), \\
\cos \left(v_{0}-\theta\right) & =\sec \lambda \cos \left(v_{1}-\theta\right) .
\end{aligned}
$$

We have for the radius vector

$$
\frac{1}{r_{1}}=\frac{1}{a_{1}\left(1-e_{1}^{2}\right)}\left\{1+e_{1} \cos \left(v_{1}-\varpi_{1}\right)\right\},
$$

and the reduced radius vector is thence found as follows: viz. we have $r_{0}=r_{1} \cos \lambda$, that is

$$
\frac{1}{r_{0}}=\frac{1}{a_{1}\left(1-e_{1}^{2}\right)}\left\{\sec \lambda+e_{1} \sec \lambda \cos \left(v_{1}-\mho_{1}\right)\right\}
$$

but $e_{1} \sec \lambda \cos \left(v_{1}-\varpi_{1}\right)$

$$
\begin{aligned}
& =e_{1} \sec \lambda \cos \left\{\left(v_{1}-\theta\right)-\left(\varpi_{1}-\theta\right)\right\}, \\
& =e_{1} \sec \lambda \cos \left(v_{1}-\theta\right) \cos \left(\varpi_{1}-\theta\right)+e_{1} \sec \lambda \sin \left(v_{1}-\theta\right) \sin \left(\varpi_{1}-\theta\right), \\
& =e_{1} \sec \lambda \cos \left(v_{1}-\theta\right) \cos \left(\varpi_{1}-\theta\right)\left\{1+\tan \left(v_{1}-\theta\right) \tan \left(\varpi_{1}-\theta\right)\right\}, \\
& =e_{0} \cos \left(v_{0}-\theta\right) \cos \left(\varpi_{0}-\theta\right)\left\{1+\tan \left(v_{0}-A\right) \tan \left(\varpi_{0}-\theta\right)\right\} \\
& =e_{0} \cos \left(v_{0}-\theta\right) \cos \left(\varpi_{0}-\theta\right)+e_{0} \sin \left(\varpi_{0}-\theta\right) \sin \left(\varpi_{0}-\theta\right), \\
& =e_{0} \cos \left(v_{0}-\varpi_{0}\right)
\end{aligned}
$$


and by the definition of $a_{0}$ we have $a_{0}\left(1-e_{0}^{2}\right)=a_{1}\left(1-e_{1}^{2}\right)$. Hence

$$
\frac{1}{r_{0}}=\frac{1}{a_{0}\left(1-e_{0}^{2}\right)}\left\{\sec \lambda+e_{0} \cos \left(v_{0}-\varpi_{0}\right)\right\}
$$

which, combined with the equation

$$
\tan \lambda=\tan \phi \sin \left(v_{0}-\theta\right)
$$

determines the position of the body in terms of the modified elements and of the reduced longitude $v_{0}$. Introducing into the two equations $s(=\tan \lambda)$ and $\gamma(=\tan \phi)$ in the place of $\lambda$ and $\phi$, they become

$$
\begin{aligned}
\frac{1}{r_{0}} & =\frac{1}{a_{0}\left(1-e_{0}^{2}\right)}\left\{\sqrt{ }\left(1+s^{2}\right)+e_{0} \cos \left(v_{0}-\varpi_{0}\right)\right\}, \\
s & =\gamma \sin \left(v_{0}-\theta\right),
\end{aligned}
$$

which is the form in which the equations occur in the lunar theory.

Proceeding now to the formulæ which involve the time, it is to be remarked that the true anomaly and the quotient of the radius vector by the semiaxis-major are given functions of the eccentricity and the mean anomaly, and calling for a moment the last-mentioned quantities $e, \xi$, I represent the functions in question by

$$
\text { elta }(e, \xi) \text {, elqr }(e, \xi) \text {, }
$$

or more simply when the mean anomaly only is attended to by

$$
\text { elta } \xi \text {, elqr } \xi \text {. }
$$

I have found this notation very convenient as a means of dispensing with the introduction of the eccentric anomaly.

The reduced longitude is found in terms of the time by means of the equations

$$
\begin{aligned}
\tan \left(v_{0}-\theta\right) & =\sec \phi \tan \left(v_{1}-\theta\right), \\
v_{1}-\varpi_{1} & =\operatorname{elta}\left(n t+\epsilon_{1}-\varpi_{1}\right),
\end{aligned}
$$

the former equation gives, as is well known,

$$
v_{0}-\theta=v_{1}-\theta-\tan ^{2} \frac{1}{2} \phi \sin \left(2 v_{1}-2 \theta\right)+\frac{1}{2} \tan ^{4} \frac{1}{2} \sin \left(4 v_{1}-4 \theta\right)-\& c .,
$$

(where the successive coefficients are the reciprocals of the natural numbers) we have therefore

$$
v_{0}=v_{1}-\tan ^{2} \frac{1}{2} \phi \sin \left\{\left(2 v_{1}-2 \varpi_{1}\right)+\left(2 \varpi_{1}-2 \theta\right)\right\}+\& c .,
$$

or, as it may be written,

$$
\begin{aligned}
v_{0}=v_{1} & -\tan ^{2} \frac{1}{2} \phi\left\{\sin \left(2 v_{1}-2 \varpi_{1}\right) \cos \left(2 \varpi_{1}-2 \theta\right)+\cos \left(2 v_{1}-2 \varpi_{1}\right) \sin \left(2 \varpi_{1}-2 \theta\right)\right\} \\
& +\frac{1}{2} \tan ^{4} \frac{1}{2} \phi\left\{\sin \left(4 v_{1}-4 \varpi_{1}\right) \cos \left(4 \varpi_{1}-4 \theta\right)+\cos \left(4 v_{1}-4 \varpi_{1}\right) \sin \left(4 \varpi_{1}-4 \theta\right)\right\} \\
& -\& c .,
\end{aligned}
$$


and for the present purpose it is only necessary to attend to the non-periodic part of the function on the right-hand side. Now

$$
v_{1}-\varpi_{1}=\operatorname{elta}\left(n t+\epsilon_{1}-\varpi_{1}\right)
$$

the non-periodic part of which is $n t+\epsilon_{1}-\varpi_{1}$. And the non-periodic part of $\frac{\cos }{\sin } \mu\left(v_{1}-\varpi_{1}\right)$ is given by the equation (62) of Hansen's Memoir "Entwickelung des Products u. s.w." Abhand. der K. Sächs. Gesellschaft zu Leipzig, t. II. (1853). In fact, Hansen's

$$
\beta \text { is }=\frac{e_{1}}{1+\sqrt{ }\left(1-e_{1}^{2}\right)},=\tan \frac{1}{2} \kappa_{1}
$$

and the formula gives for the non-periodic parts

$$
\begin{aligned}
& \cos \mu\left(v_{1}-\sigma_{1}\right)=(-)^{\mu} \tan ^{\mu} \frac{1}{2} \kappa_{1}\left(1+\mu \cos \kappa_{1}\right), \\
& \sin \mu\left(v_{1}-\sigma_{1}\right)=0 .
\end{aligned}
$$

Hence, substituting these values and putting for the non-periodic part of $v_{0}$ the assumed value $n t+\epsilon_{0}$, we find

$$
\begin{aligned}
\epsilon_{0}=\epsilon_{1} & -\tan ^{2} \frac{1}{2} \phi \tan ^{2} \frac{1}{2} \kappa_{1}\left(1+2 \cos \kappa_{1}\right) \sin \left(2 \sigma_{1}-2 \theta\right) \\
& +\frac{1}{2} \tan ^{4} \frac{1}{2} \phi \tan ^{4} \frac{1}{2} \kappa_{1}\left(1+4 \cos \kappa_{1}\right) \sin \left(4 \varpi_{1}-2 \theta\right) \\
& -\& c .
\end{aligned}
$$

The series on the right-hand side may be summed without difficulty, and we obtain

$$
\begin{aligned}
\epsilon_{0}=\epsilon_{1} & -\tan ^{-1}\left\{\frac{\tan ^{2} \frac{1}{2} \phi \tan ^{2} \frac{1}{2} \kappa_{1} \sin \left(2 \sigma_{1}-2 \theta\right)}{1+\tan ^{2} \frac{1}{2} \phi \tan ^{2} \frac{1}{2} \kappa_{1} \cos \left(2 \sigma_{1}-2 \theta\right)}\right\} \\
& -2 \cos \kappa_{1} \frac{\tan ^{2} \frac{1}{2} \phi \tan ^{2} \frac{1}{2} \kappa_{1} \sin \left(2 \sigma_{1}-2 \theta\right)}{1+2 \tan ^{2} \frac{1}{2} \phi \tan ^{2} \frac{1}{2} \kappa_{1} \cos \left(2 \sigma_{1}-2 \theta\right)+\tan ^{4} \frac{1}{2} \phi \tan ^{4} \frac{1}{2} \kappa_{1}},
\end{aligned}
$$

in which formula the values of $\tan \frac{1}{2} \phi, \tan \frac{1}{2} \kappa_{1}$ (in terms of $\gamma, e_{1}$ ) are

$$
\frac{\gamma}{1+\sqrt{ }\left(1+\gamma^{2}\right)}, \quad \frac{e_{1}}{1+\sqrt{ }\left(1-e_{1}^{2}\right)},
$$

and that of $\cos \kappa_{1}$ is $\sqrt{ }\left(1-e_{1}^{2}\right)$. We have thus the required expression for the modified mean longitude at epoch, and all the modified elements are now expressed in terms of the original elements.

The following investigation leads to a theorem which it is, I think, worth while to notice. We have

$$
r_{0}^{2} \frac{d v_{0}}{d t}=\sqrt{ }\left\{\sigma a_{0}\left(1-e_{0}^{2}\right)\right\} \cos \phi
$$


and thence

$$
\begin{aligned}
d t & =\frac{a_{0}^{\frac{3}{2}}\left(1-e_{0}^{2}\right)^{\frac{3}{2}} d v_{0}}{\sqrt{(\sigma) \cos \phi}\left\{\sec \lambda+e_{0} \cos \left(v_{0}-\varpi_{0}\right)\right\}^{2}} \\
& =\frac{a_{1}^{\frac{3}{2}}\left(1-e_{1}^{2}\right)^{\frac{3}{2}} d v_{0}}{\sqrt{ }(\sigma) \sqrt{ }\left(1+\gamma^{2}\right)\left[\sqrt{ }\left\{1+\gamma^{2} \sin ^{2}\left(v_{0}-\varpi_{0}\right)\right\}+e_{0} \cos \left(v_{0}-\varpi_{0}\right)\right]^{2}}
\end{aligned}
$$

or as it may be written

$$
\begin{aligned}
\frac{d v_{0}}{\left[\sqrt{ }\left\{1+\gamma^{2} \sin ^{2}\left(v_{0}-\varpi_{0}\right)\right\}+e_{0} \cos \left(v_{0}-\varpi_{0}\right)\right]^{2}} & =\sqrt{\left(\frac{\sigma}{a_{1}^{3}}\right)\left(1-e_{1}^{2}\right)^{-\frac{3}{2}}\left(1+\gamma^{2}\right)^{\frac{1}{2}} d t} \\
& =\quad n\left(1-e_{1}^{2}\right)^{-\frac{3}{2}}\left(1+\gamma^{2}\right)^{\frac{1}{2}} d t .
\end{aligned}
$$

But it is easy to see that if the mean longitude $n t+\epsilon_{0}$ is expanded in terms of $v_{0}$, the relation between these quantities must be of the form $n t+\epsilon_{0}=v_{0}+$ periodic terms. It follows that in the preceding equation the non-periodic part of the function which multiplies $d v_{0}$ (the expansion being in multiple cosines of $v_{0}$ ) must be equal to $\left(1-e_{1}^{2}\right)^{-\frac{8}{2}}\left(1+\gamma^{2}\right)^{\frac{1}{2}}$. Hence, putting for $e_{1}$ its value, we find that the non-periodic part of

$$
\frac{1}{\left[\sqrt{ }\left\{1+\gamma^{2} \sin ^{2}\left(v_{0}-\theta\right)\right\}+e_{0} \cos \left(v_{0}-\varpi_{0}\right)\right]^{2}},
$$

expanded in multiple cosines of $v_{0}$ is

$$
\left[1-e_{0}^{2}\left\{1-\frac{\gamma^{2}}{1+\gamma^{2}} \sin ^{2}\left(\varpi_{0}-\theta\right)\right\}\right]^{-\frac{3}{2}}\left(1+\gamma^{2}\right)^{\frac{1}{2}},
$$

a theorem which might, it is probable, be verified without much difficulty.

2, Stone Buildings, October, 1856. 